\title{
Transanal minimally invasive surgery (TAMIS) for rectal tumor: a case report and literature review
}

\author{
Huipeng Wang", Tao Ye", Jun Chen, Lifeng Gong, Wenjie Chen, Jiamen Shen, Jiaying Zhao*, \\ Yuankun Cai* \\ Department of General Surgery, The Fifth People's Hospital of Shanghai, Fudan University, Shanghai, China \\ "These authors contributed equally to this work as co-first authors. \\ *These authors contributed equally to this work as co-correspondence authors. \\ Correspondence to: Yuankun Cai, MD; Jiaying Zhao. Chief Surgeon of General Surgery, Associate Professor, The Fifth People's Hospital of Shanghai, \\ Fudan University, 801 Heqing Road, Shanghai, China. Email: yuankun@medmail.com.cn; zhaojiaying001@126.com.
}

\begin{abstract}
Transanal minimally invasive surgery (TAMIS) is mainly used for benign tumors of the rectum, but there are few reports on treating malignant tumors of the rectum with a large volume. The aim of this study was to evaluate the feasibility and safety of TAMIS for resection of rectal malignant tumors. A 57-yearold patient was pathologically diagnosed as rectal malignancy before surgery. Computed tomography (CT), magnetic resonance imaging (MRI), fludeoxyglucose-18F (18F-FDG), and endoscopic mucosal biopsy were performed to assess the tumor location and preoperative size. There were no contraindications in all preoperative examinations. We applied TAMIS technology to perform the operation on this patient, And the operative time, the amount of blood loss, the length of hospital stay, the cost of hospital stay, surgical complications, postoperative complications and other relevant data were all collected. The operation was successful, the operation time was $45 \mathrm{~min}, 10 \mathrm{~mL}$ of intraoperative blood loss, and the length of stay was 3 days, a tumor of a maximum diameter of $4 \mathrm{~cm}$ being completely removed. There were no related complications or recurrence during postoperative follow-up. The pathological results were tubular villous adenoma with low-grade intraepithelial neoplasia, and a focal area of high-grade intraepithelial neoplasia. The pathological stage was T1N0M0. At 3-month follow-up there were no signs of recurrence. The patient was followed up for 5 years after the operation and there was no tumor recurrence or metastasis in other parts and no other discomfort. TAMIS is less commonly used in rectal malignancies, especially for tumors with larger diameters. We successfully performed complete resection of a 4-cm rectal malignant tumor with TAMIS. Given its low risk, low cost, simple operation, and few complications, TAMIS can be used for more indications of rectum diseases.
\end{abstract}

Keywords: Transanal minimally invasive surgery (TAMIS); rectal cancer; laparoscopic surgery

Submitted May 12, 2020. Accepted for publication Jul 13, 2020.

doi: $10.21037 /$ atm-20-4346

View this article at: http://dx.doi.org/10.21037/atm-20-4346

\section{Introduction}

Traditional transanal surgery is only used to treat anal lesions which the distance within $6 \mathrm{~cm}$ of the anal margin. Therefore, the treatment of tumors in a high position of the rectum has often only included colonoscopy and submucous resection (ESR or ESD). Tumors treated this way are often not completely resected or are broken, and full intestinal wall thickness resection is not possible. Faced with this embarrassing situation, some patients have to accept a laparoscopic-assisted or open colorectal surgery. This kind of operation entails great trauma, many surgical complications, high hospitalization cost, and long hospitalization time. Transanal excision (TAE) is performed with an anal retractor, but when the position of the tumor is high in the rectum, this procedure is challenging and 


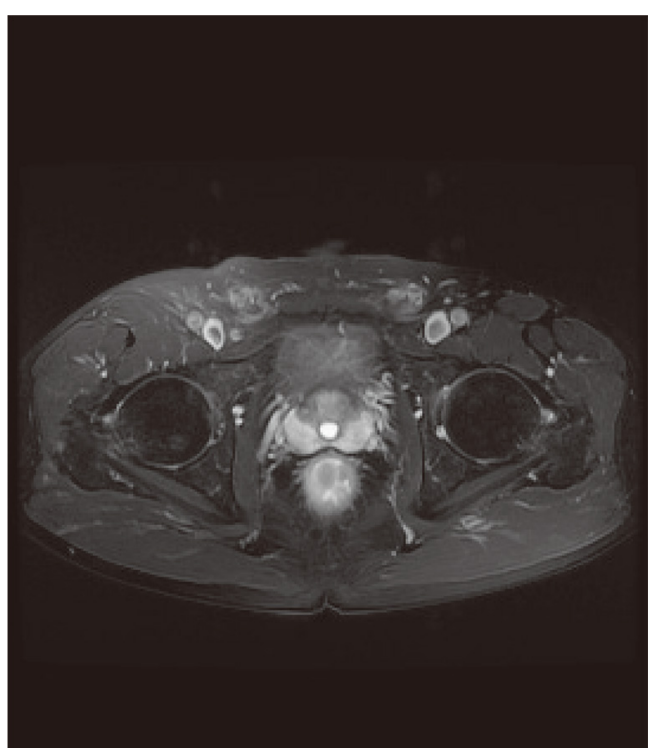

Figure 1 Location of the tumor. MRI shows a mass in the rectum, indicating a tumor. MRI, magnetic resonance imaging.

the local recurrence rate is high. Dr. Gerhard Buess first proposed forward transanal endoscopic microsurgery (TEM) in 1983, and, although TEM greatly improved the situation, the system's instruments are expensive, the operation assembly is complex, and the learning curve is steep (1-3).

In 2009, Atallah et al. combined TEM with singleport laparoscopic technology and proposed an innovative method of transanal minimally invasive surgery (TAMIS); that is, through the application of common laparoscopic equipment, a surgical effect similar to that of TEM can be achieved at a lower cost. Since TAMIS is no longer limited by specialized proctoscopies and instruments, it can be applied to a wider range of lesions and indications (4). TAMIS can be used for the treatment of benign rectal tumors, early rectal cancer (stage T1), and submucosal tumors, as well as advanced rectal cancer (stage $\mathrm{T} 2$ and T3) with many systemic complications that cannot tolerate major surgery, as well as rectal stenosis, anastomotic fistula, rectovaginal fistula and other diseases. It avoids enterotomy and is minimally invasive. It is especially suitable for elderly patients.

The gastrointestinal surgery department of our hospital completed the first TAMIS operation in Shanghai in September 2014. And the tumor is malignant of the rectum with a large volume of $4 \mathrm{~cm}$ diameter. Besides, we improved the traditional TAMIS platform with an airbag in the device. Therefore, as the first department to perform TAMIS surgery in Shanghai, we here report a case of rectal malignant tumor treated by TAMIS with 5 -year followup. We present the following case in accordance with the CARE reporting checklist (available at http://dx.doi. org/10.21037/atm-20-4346).

\section{Case presentation}

\section{Patient data}

A 57-year-old male was admitted to the Department of General Surgery on March 16, 2015, due to "Defecation deformity for more than one year and aggravation for 2 weeks". Two weeks prior, he had visited the First Hospital of Ningbo in Zhejiang province for treatment. Colonoscopy (No. 201500001214) indicated a large lateral mass 4$8 \mathrm{~cm}$ from the anal margin, occupying the intestinal cavity for about half a week, and a generally normal remaining intestinal mucosa. Pathology (Clinical Pathological Diagnosis Center of Ningbo No. GF2015-013808) revealed villous adenoma with low-grade intraepithelial neoplasia, with a focal area of high-grade intraepithelial neoplasia. The patient denied a history of diseases such as hypertension and diabetes, as well as a history of genetic diseases such as tumors.

Physical examination yielded the following: body temperature, $36.2{ }^{\circ} \mathrm{C}$; heart rate, 80 beats per minute; breath, 19 beats per minute; blood pressure, 125/80 mmHg; height, $164 \mathrm{~cm}$; and weight, $65 \mathrm{~kg}$. Meanwhile, the digital rectal examination results were the following: 1-6 o'clock in the knee-chest $(\mathrm{KC})$ position, about $5 \mathrm{~cm}$ from the anal margin, a $3 \times 4 \mathrm{~cm}^{2}$ mass growing prominent in the intestinal cavity, with soft, mobile, unclear edges, and no tenderness or bloodstaining.

\section{Preoperative preparation}

Computed tomography (CT), magnetic resonance imaging (MRI) (Figure 1), fludeoxyglucose-18F (18F-FDG), and endoscopic mucosal biopsy were performed to assess the tumor location and preoperative size, and ruled out advanced malignancy, local lymph node metastasis, and other metastasis. Cardiopulmonary function and anesthesia risk were also assessed. Three days before the operation, oral administration of cefradine and metronidazole antibiotics was given. Low-residue, liquid diet, intestinal preparation by polyethylene glycol electrolyte powder was provided 


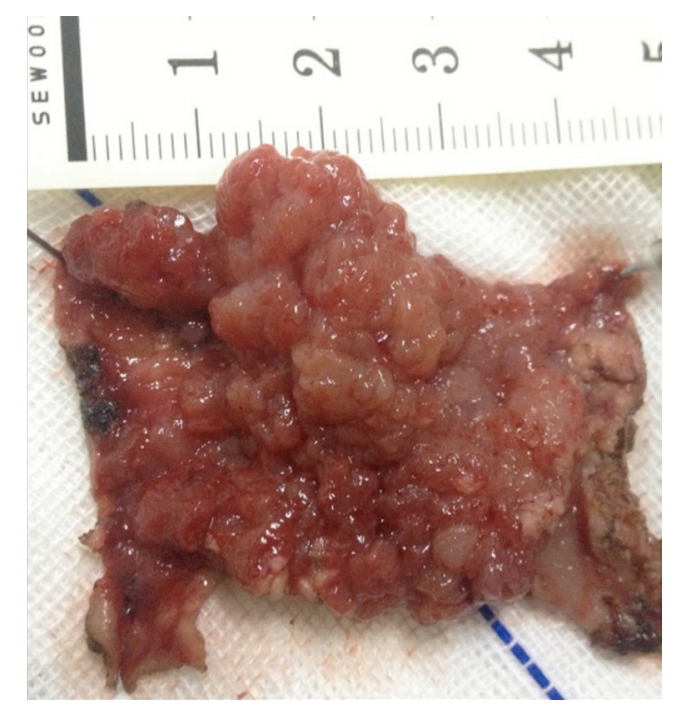

Figure 2 Pathological specimen. Pathological data show a tumor $4 \times 2.5 \times 2 \mathrm{~cm}^{3}$ in size.

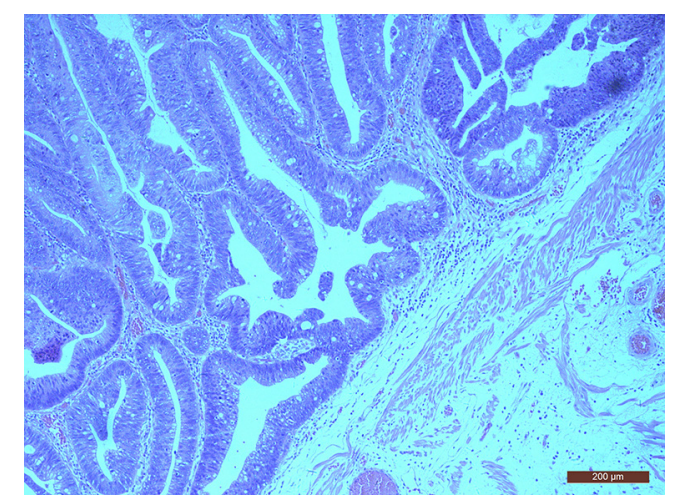

Figure 3 Histopathological image of the tissue showing tubular villous adenoma.

1 day before the operation. Fasting for 8 hours before the operation and refraining from drinking 6 six hours before the operation were required.

\section{Surgical procedure}

General anesthesia was implemented. With the patient in the knee-chest position, a gauze was placed into the rectum at the proximal lesion about $10 \mathrm{~cm}$ to close the rectum to stop discharge and wastewater. A SILS port (Covidien, Mansfield, MA, USA) was fixed to the anus, and $\mathrm{CO} 2$ pressure was maintained at $15 \mathrm{mmHg}$ and $12 \mathrm{~L} / \mathrm{min}$. The tumor was located at 1-6 o'clock and was about $3 \times$
$4 \mathrm{~cm}^{2}$ in size, with a soft, smooth surface, and wide base of approximately $4 \times 4 \mathrm{~cm}^{2}$. A circular incision was made $0.5 \mathrm{~cm}$ from the tumor edge by hook. And then continue to cut deep into the mucosal layer, submucosa, or muscle layer gradually, depending on the depth of the lesion, until complete resection was achieved (Figure 2). The wound was closed and an anal canal was placed for drainage.

\section{Postoperative treatment and results}

On the first day after the operation, fasting and total parenteral nutrition were given. On the second day, a fluid diet was given, and the anal canal was removed. Intravenous cefmeazole was administered 24 hours after the operation to prevent infection. He was discharged from the hospital on the third day after surgery. There were no obvious postoperative complications, such as urinary retention, urinary tract infection, blood stool, stool incontinence, intestinal obstruction, etc.

The pathological results were as follows: a tumor diameter of $4 \mathrm{~cm}$, tubular villous adenoma with low-grade intraepithelial neoplasia, and a focal area of high-grade intraepithelial neoplasia (Figure 3). There was no tumor at the basement and cutting edge. The pathological stage was T1N0M0.

\section{Follow-up}

At 3-month follow-up there were no signs of recurrence. The patient was followed up for 5 years after the operation, and a digital rectal examination was carried out. There was no tumor recurrence or metastasis in other parts and no other discomfort.

All procedures performed in this case involving human participants were in accordance with the Declaration of Helsinki (as revised in 2013). Written informed consent was obtained from the patient.

\section{Discussion}

The initial indications for TAMIS surgery are benign tumors in the rectum and early malignant tumors (stage T1-T2). The optimal surgical range for TAMIS is $5-18 \mathrm{~cm}$. However, the application of TAMIS has been expanded to treat neuroendocrine tumors, low rectal anastomotic fistula, rectourethral fistula, removal of high rectal foreign bodies, and other conditions $(5,6)$. Besides this, robot-assisted TAMIS, TAMIS-TME technology, TAMIS-laparoscopic 
combined technology, and endoscope-assisted TAMIS (eTAMIS) $(7,8)$ have also been rapidly developed.

In our study, although the patient had a large tumor, the operation was completed without resorting to laparotomy, endoscopic local mucosal resection, or laparoscopic-assisted surgery, indicating the feasibility of TAMIS surgery for rectal tumor resection. During the operation, we found that the surgical field of vision was not a closed space, and the unstable air pressure during the operation had a certain impact on the surgical field of vision. We applied a tip to fill a piece of gauze to the proximal end of the intestinal tract so that the air pressure was relatively stable during the operation and the surgical field was exposed clearly. In this case, the operation time was $45 \mathrm{~min}$, $10 \mathrm{~mL}$ of intraoperative blood loss, and the average length of stay was 3 days, all of these had greater advantages than the conventional open surgery, which reduced the intraoperative complications and economic burden of the patient, and is undoubtedly a promising result for patients with benign rectal tumors and early malignant tumors. Unlike with traditional open surgery, there were no obvious complications (urinary retention, blood in the stool, fecal incontinence, intestinal obstruction, etc.) in the patients after the operation. The patients were followed up for 3 months to 5 years without recurrence.

Although TAMIS is operated through the natural channel and provides minimal damage to the patient, rapid recovery, low cut edge-positive rate, and lower recurrence rate and mortality. There are also some disadvantages in this case, unlike laparoscopic surgery, the rectal space is limited and the operation is somewhat difficult. Compared with large tumors, complete resection is not possible. Besides, there are still some questions worth considering further.

(I) Does TAMIS have a negative effect on the anal sphincter in relation to the SILS port and TAMIS surgery time? The SILS port has a diameter of 3.5-4 $\mathrm{cm}$, is soft and elastic, can adapt to the shape of anus, and protects the function of anal sphincter. The short-term postoperative follow-up of the patients we collected showed no anal incontinence or other related complications, and other studies have also indicated that the port had no significant effect on the function of the anus. In the modern era, patients' quality of life is highly valued, and it is thus necessary to allow patients to retain normal anal function. We should therefore determine which factors in the TAMIS approach, such as operation duration, operation mode, port material properties, etc., influence anal function, so that clinicians can make preparations for optimizing patient quality, standardizing surgical operation, and achieving better treatment effect in the future.

(II) How can a stable intestinal pressure be established to ensure a successful operation? Bislenghi et al. successfully completed TAMIS with the airseal system $(9,10)$. On the basis of follow-up studies, we inserted an air bag into the rectum, which could guarantee the stability of air pressure to a certain extent.

(III) Does TAMIS for rectal malignancy increase the rates and incidence of infection, anastomotic fistula, tumor implantation, and pelvic recurrence? Currently, the answer to these questions remain unclear. Different institutions have different results. Haugvik et al. studied 51 patients who underwent TAMIS at a single institution from 2011 to 2015 were retrospectively. Overall morbidity was $12 \%$. $22 \%$ had a positive resection margin, whereas $31 \%$ had an indefinable resection margin status mostly due to tissue fragmentation (11).

(IV) If the surgical wound is not closed, does it increase the risk of bleeding and related complications? The amount of intraoperative blood loss in TAMIS is small, but suturing the operation wound is a difficult and lengthy procedure. Hahnloser conducted a small-scale clinical control study on this, and found that there was no significant difference in the occurrence of postoperative bleeding complications caused by wound opening or suture (12). Of course, these problems need to be further discussed in a large-scale, standardized, clinical practice study.

There are several recommendations for TAMIS surgery. First of all, strict screening of suitable cases, for rectal benign tumors, the diameter of the base should not be too large, or it is difficult to remove. Secondly, in the case of rectal malignant tumor, $\mathrm{T} 1$ or early $\mathrm{T} 2$ tumor patients with small volume should be selected as far as possible without metastasis. Preoperative MRI is helpful to determine the depth of tumor invasion before surgery, ensure the integrity of resection and negative resection margins.

Overall, our experience with TAMIS has led us to conclude that it is a reliable technique for the treatment of benign tumors and early malignant tumors of the rectum, but it still requires further standardization. 


\section{Acknowledgments}

Funding: This study was supported by the Science and Technology Commission of Shanghai Municipally (No. 17411967600), Natural Science Research Funds of Minhang District, Shanghai (No. 2018MHZ025) and Natural Science Research Funds of Minhang District, Shanghai (No. 2018MHZ037).

\section{Footnote}

Reporting Checklist: The authors have completed the CARE reporting checklist. Available at http://dx.doi.org/10.21037/ atm-20-4346

Conflicts of Interest: All authors have completed the ICMJE uniform disclosure form (available at http://dx.doi. org/10.21037/atm-20-4346). The authors have no conflicts of interest to declare.

Ethical Statement: The authors are accountable for all aspects of the work in ensuring that questions related to the accuracy or integrity of any part of the work are appropriately investigated and resolved. All procedures performed in this case involving human participants were in accordance with the Declaration of Helsinki (as revised in 2013). Written informed consent was obtained from the patient.

Open Access Statement: This is an Open Access article distributed in accordance with the Creative Commons Attribution-NonCommercial-NoDerivs 4.0 International License (CC BY-NC-ND 4.0), which permits the noncommercial replication and distribution of the article with the strict proviso that no changes or edits are made and the original work is properly cited (including links to both the formal publication through the relevant DOI and the license). See: https://creativecommons.org/licenses/by-nc-nd/4.0/.

\section{References}

1. Mclemore EC, Weston LA, Coker AM, et al. Transanal

Cite this article as: Wang H, Ye T, Chen J, Gong L, Chen W, Shen J, Zhao J, Cai Y. Transanal minimally invasive surgery (TAMIS) for rectal tumor: a case report and literature review. Ann Transl Med 2020;8(17):1101. doi: 10.21037/atm-20-4346 minimally invasive surgery for benign and malignant rectal neoplasia. Am J Surg 2014;208:372-81.

2. Barendse RM, Dijkgraaf MG, Rolf UR, et al. Colorectal surgeons' learning curve of transanal endoscopic microsurgery. Surg Endosc 2013;27:3591-602.

3. Maya A, Vorenberg A, Oviedo M, et al. Learning curve for transanal endoscopic microsurgery: a single-center experience. Surg Endosc 2014;28:1407-12.

4. Atallah S, Albert M, Larach S. Transanal minimally invasive surgery: a giant leap forward. Surg Endosc 2010;24:2200-5.

5. Cahill RA. Transanal Total Mesorectal Excision: The Next 10 Years. In: Transanal Minimally Invasive Surgery (TAMIS) and Transanal Total Mesorectal Excision (taTME). 2019.

6. Lee L, Althoff A, Edwards K, et al. Outcomes of Closed Versus Open Defects After Local Excision of Rectal Neoplasms: A Multi-institutional Matched Analysis. Dis Colon Rectum 2018;61:172-8.

7. Hompes R, Rauh SM, Ris F, et al. Robotic transanal minimally invasive surgery for local excision of rectal neoplasms. Br J Surg 2014;101:581.

8. Wexner SD, Berho M. Transanal TAMIS total mesorectal excision (TME) - a work in progress. Tech Coloproctol 2014;18:423-5.

9. Mclemore EC, Coker A, Jacobsen G, et al. eTAMIS: endoscopic visualization for transanal minimally invasive surgery. Surg Endosc 2013;27:1842-5.

10. Bislenghi G, Wolthuis AM, de Buck van Overstraeten A, et al. AirSeal system insufflator to maintain a stable pneumorectum during TAMIS. Tech Coloproctol 2015;19:43-5.

11. Haugvik SP, Groven S, Bondi J, et al. A critical appraisal of transanal minimally invasive surgery (TAMIS) in the treatment of rectal adenoma: a 4-year experience with 51 cases. Scand J Gastroenterol 2016;51:855-9.

12. Hahnloser D, Cantero R, Salgado G, et al. Transanal minimal invasive surgery for rectal lesions: should the defect be closed? Colorectal Dis 2015;17:397-402 . 